Voix et Images

volxetimages

\title{
Gérard Bessette et la recherche du Père
}

\section{Jean Fisette}

Volume 1, numéro 3, avril 1976

\section{Gérard Bessette}

URI : https://id.erudit.org/iderudit/200034ar

DOI : https://doi.org/10.7202/200034ar

Aller au sommaire du numéro

Éditeur(s)

Les Presses de l'Université du Québec

ISSN

0318-9201 (imprimé)

1705-933X (numérique)

Découvrir la revue

Citer ce document

Fisette, J. (1976). Gérard Bessette et la recherche du Père. Voix et Images, 1(3), 317-328. https://doi.org/10.7202/200034ar d'utilisation que vous pouvez consulter en ligne.

https://apropos.erudit.org/fr/usagers/politique-dutilisation/ 


\section{Gérard Bessette et la recherche du Père}

NDLR: Nous avons été très amicalement reçus par Gérard Bessette, à son appartement montréalais et c'est durant tout un après-midi que s'est déroulé cet entretien dont je présente ici un compte rendu. Je remercie Jacques Allard, Alain Piette et André Vanasse avec qui cette entrevue a été réalisée. (Jean Fisette)

V.I. Gérard Bessette, vous avez une double pratique d'écrivain d'imagination et de critique. Vous avez déjà dit ${ }^{1}$ que vous réussissez à établir une coupure nette entre ces deux pratiques. Qu'en pensez-vous maintenant?

G. B. J'essaie de ne pas penser à la psychanalyse, ni'à mes connaissances littéraires, quand j'écris le premier jet. Je me rends bien compte que, quand je corrige, je me sers de mes connaissances théoriques; mais quand je plonge dans le premier jet, dans le brouillon, j'essaie d'écarter de mon esprit, toutes mes connaissances. On ne peut jamais anniniler ces choses-là; mais on peut les repousser, les refouler. J'ai l'impressıon, en tous cas, que si je n'établissais pas cette séparation entre les deux domaınes, cela me paralyserait.

V.I. Alors, est-ce que le Cycle constituerait une exception par rapport aux autres œuvres? J'entends les procédés, fort calculés, marqués par les jeux de parenthèses et qui présupposent la connaissance de la psychanalyse, comme l'a souligné R. Robidoux ${ }^{2}$ ?

G. B. Vous parlez naturellement de l'œuvre termınée et non pas de l'œuvre "se faișant", comme aurait dit Bergson. L'emploi particulier des tırets et des parenthèses peut paraitre "calculé " aux yeux du lecteur. II ne m'appartient pas d'en juger. Seulement il me faut souligner que cette ponctuation singulière n'était pas préméditée. Elle s'est produite en cours de route. A un certain moment je me suis aperçu que j'avais tendance à employer le tiret pour tel genre de notation et la parenthèse pour tel autre, d'une façon d'alleurs irrégulière et tâtonnante. C'est seulement dans la deuxième ou la troisième version que j'ai tenté d'uniformiser le "procédé". 
Il en va de même pour les ellipses. Je me suis rendu compte à la relecture que plusieurs verbes "manquaient». Ces tournures elliptiques m'ont paru propices à l'expression du monologue intérieur et j'ai ensuite pratıqué de nouvelles "coupures"...

V.I. Pourrait-on voir un rapport entre le récit à la première personne et l'emploı de la parenthèse? De fait, il est notable que dans les deux romans écrits à la troisı̀me personne (les Pédagogues et la Bagarre), il y ait absence presque totale de la parenthèse, et surtout pas de ce type de parenthèse qui se veut explicatıf, maıs qui double le texte, avec une certaine ironie d'ailleurs?

G. B. Oui, je crois que chez mol, l'emploi du JE romanesque a coïncıdé en gros avec la "découverte" de la complexité de la psyché. Je me suis souvenu que Freud (et Mélanie Klein) ont démontré que l'être humain était multiple avant de devenir un. Au début, if y a indifférenciation, "a-dualisme" si vous voulez. Le moi émerge lentement, péniblement d'un chaos primordial. L'enfant n'a pas de JE au sens adulte. Avant de me rendre compte de notre multiplicité psychique originaire, j'étais très exıgeant - peut-être trop rigide - sur le chapitre de l'optique romanesque. Dans certaines de mes études sur Gabrielle Roy ${ }^{3}$, je lui reproche carrément ses changements d'optique, ses interventions d'auteur. Aujourd'hui je serais moins catégorique même si je trouve encore que Gabrielle Roy n'a pas toujours été optiquement habile.

Maintenant je me dis que si le romancier omniscient a régné si longtemps (à son insu et à l'insu du lecteur), c'est que son attitude démiurgique et "ubiquiste" devait correspondre à une tendance profonde, universelle. C'est parce que nous avons été "polyfocaux" durant notre enfance que, une fois adultes, nous acceptons si facilement les changements d'optique romanesque.

On a beau dire qu'il s'agit d'une convention littéraire, d'une habitude, etc., l'explication est insuffisante. Chacun de nous est optiquement le centre du monde: voilà la vérité, la réalité. Les optiques multiples, voire sımultanées de l'auteur omniscient n'auraient pas si facılement passé la rampe si l'enfant ne s'était pas identifié successivement et/ou simultanément avec ses parents, s'il n'avait pas vu le monde par leurs yeux... Voilà ce que j'ai découvert: le phénomène infantile et spontané de l'ıdentification. J'appelle ça une découverte... Au fond, personne ne découvre jamaıs rien. Mais ce qu'on savait déjà d'une façon abstraite ou ımplicite devient tout à coup du vécu psychique, organique...

V.I. Vous dites que lors du premier jet, vous plongez, vous entrez dans une sorte de transe; et qu'ensuite, vous polissez, vous retravallez, etc. Pourriez-vous préciser le chemınement de vos technıques de travail lorsque vous écrivez un roman?

G. B. "Transe", c'est un peu fort comme terme; je préférerals "état second". Je me rends compte de plus en plus que je suis à peu près Incapable de fantasmer sans avoir un crayon à la main. Et j'ai bien l'impres- 
sion que je continuerais à écrire, même s'il n'y avait aucune possibilité de publication, parce que c'est le crayon qui me rattache à l'adolescence et à l'enfance. A mesure que l'on vieillit, Il devient de plus en plus difficile de fantasmer, à moıns d'avoir un truc, un procédé pour le farre.

\section{V.1. Et comment y arrivez-vous?}

G. B. Je n'ai pas tellement de technıque, sauf que lorsque je n'enseigne pas, je passe tous les jours un minimum de deux heures - souvent c'est quatre - un crayon à la main.

V.I. Vous repolıssez très longtemps, si l'on se fie à ce quı est arrivé à la Commensale?

G. B. J'écris parfoıs très lentement. Je dis “fantasmer", maıs ça ne vient pas toujours sı facilement. Quand je ne peux pas démarrer, je feullette un dictıonnaıre analogique; c'est un des meilleurs tremplins que je connaisse. Ce dictionnarre me fournit des constellations de mots qui me permettent souvent de démarrer. Une autre façon de travaıller pour que le démarrage ne soit pas trop lent, c'est de ne pas se vider complètement la veille, en prévision du lendemain: lassser encore quelque chose à dire, jeter un ou deux mots sur le papıer comme aide-mémoire. Ensuite on revoit ça le lendemain et on peut repartir.

V. I. En quelque sorte, votre démarche est tout a fait à l'ınverse de celle d'Hubert Aquin qui lui, prétend-il ${ }^{4}$, fait d'abord un plan très détaillé; ensuite, et c'est l'étape finale, il entre dans une sorte de transe où tout en suivant son plan, Il peut laısser toute liberté au texte qui se fait. Vous ne croyez pas cela?

G. B. Je n'al aucune rasson de douter de ce qu'll dit. Seulement, chez lui, la scission, le dédoublement ad infinitum du personnage est à ce point constant et fondamental qu'il pourrait employer n'importe quelle technique secondaire: cela ne changerait guère la nature de ses romans.

V.I. Qu'est-ce qui est arrivé à la Commensale? Pourquol ce roman a-t-il tant tardé à paraître?

G. B. La raison qui me vient à l'esprit, c'est qu'il y a un passage “nouveau roman" qui décrit un fantasme infantile du héros et qui diffère tout à fait du reste du texte ${ }^{5}$. J'ai dû sentır le besoin de donner à Chayer un passé, des racines susceptibles de nous éclairer sur l'orıgine de ses ıdıosyncrasıes. Alors j'ai fait ce "flashback". Ensuite, je me suis dit (à cause de mon «impénitence" réaliste) qu'il était peu vraisemblable que Chayer eût un seul "flashback"; qu'il faudrait en ajouter d'autres. Mais lorsque j'ai voulu remettre la Commensale sur le métier quelques années plus tard, elle s'était considérablement refroıdıe. Je me suis sentı ıncapable de la remanier: elle auraıt éclaté. J'auraıs été obligé de la récrıre. J'avais trimé sur ce manuscrit plus que sur tout autre, au point de m'en dégoûter presque... Voilà pourquoi sans doute il est resté ce qu'il est et a eu sa drôle de naissance tardive... 
V.I. Est-ce que la ressemblance entre Chayer et Jodoın n'aurait pas joué?

G. B. Peut-être aussı.

V.I. Même sı la trame du roman est différente, les personnages se ressemblent.

G. B. Peut-être que ça a joué. Parce que j'avaıs peur de ne plus sortır du Lıbraire; quı m'agace. On me parle trop du Lıbraıre à mon goût, et pas assez des autres romans; c'est celui qui est, de loın, le plus connu. Our, ça a pu jouer.

Gilles Marcotte m'a fait une remarque récemment; il va publier bıentôt un livre sur quatre romanciers: M.-C. Blais, J. Godbout, R. Ducharme et mol. Quand je l'al vu, il m'a dit qu'en un sens, Chayer était un antıJodoin. Alors que Jodoin ne livre pas ses sentiments, Chayer, luı, laisse sa langue l'emporter: l'un a opté pour le refoulement, l'autre pour le déroulement, au plan linguistique.

V. I. Alors qu'on sent Jodoın comme le maître, le père de l'écriture - qui, de fart, figure comme une activité intégrée dans son emplor du temps Chayer paraît dépassé par le texte.

G. B. Ce que j'ai compris dans l'analyse verbale de Marcotte, c'est qu'll se plaçaıt, pour affırmer cela, non sur le plan de l'écriture, mais sur le plan du comportement des personnages.

V.I. Dans l'hıstoıre racontée ? . ........

G. B. C'est ça, ouı.

V.I. De fait, le personnage de Lagarde (dans l'Incubation) seralt à rapprocher de Jodoın et de Chayer; mais alors que Jodoın fait preuve d'une obsessıon de tout évaluer numériquement, Chayer est mathématıcien (il semble très versé, ça semble très savant!).

G. B. Ah! les équations qui notent la chute d'Athanase sont correctes; c'est un mathématıcıen quı me les a fournies!

V.I. Lagarde cherche la même précısıon au niveau du vocabulaire; les synonymes se survent comme s'il cherchait toujours plus de justesse dans la façon de cerner une réalıté. D'où le nombre consıdérable de parenthèses quı répondent justement à cette manie de la précısıon... Alors que Jodoın et Chayer paraıssent sûrs d'eux, Lagarde, lui, semble Indécıs, inquiet...

G. B. Je pense que c'est une remarque très juste et très sagace en même temps: les deux démarches, en apparence contraıres, Indiquent dans le fond, une préoccupation unıque. Ça me rappelle une partıcularıté dont je parle actuellement dans mes cours. Je travaille sur le roman en JE. (Vous savez, c'est mon projet qui remonte aux années 20 !) J'al étudié l'Aquarıum et Prochaın Épısode: je vals étudier Kamouraska, qui est un roman en ELLE-JE. Les narrateurs de l'Aquarium et de Prochain Épıso- 
de n'ont pas de nom, n'ont même pas de prénom, rien. Alors qu'à l'autre extrême, l'héroïne de Kamouraska en a troıs: elle se marie deux foıs. Et il y a là tout un jeu nominal et pronominal. Alors je pense qu'on peut aussi, en retenant les contrastes, dire qu'il y a une recherche de l'identıté qui se traduit, d'un côté par l'anonymat, de l'autre par une pléthore de noms. La remarque que vous faites est un peu de même nature; c'est une des bonnes remarques qui m'aient été faites sur mon cuvre.

V.I. Croyez-vous à la possibılité d'établir une typologıe caractérielle du phénomène littéraire (au nıveau de l'écriture plus que de l'auteur)? Par exemple, l'écriture chez Hubert Aquin relève de mécanismes schızoïdes; chez V.-L. Beaulieu, c'est un art romanesque paranoié ; chez Gilbert Laroque, c'est du type obsessionnel.

D'autre part, vous faites montre, dans vos textes critiques, d'un constant soucl esthétique. Aussı vous reprochez à certains écrivains des «erreurs"; aınsi, à V.-L. Beaulieu, dans son Jos Connalssant, de s'ımmiscer, à tıtre d'auteur, dans le texte. II semble y avoır chez vous une scission nette entre le souci esthétrque et la méthode que vous pratiquez.

Sı l'on arrıait à établir cette typologie caractérielle des textes, croyezvous que l'on pourrait en tirer une esthétique propre à chacun de ces types? Par exemple, la schizophrénie entraîne une pétarade métaphorique où les mots n'existent que par eux-mêmes; chez Ducharme, il y a le même plaisır du mot...

G. B. Cela me paraît des plus éclairants. Chez moi, ce serait plutôt du type obsessionnel. Oui, parce qu'il y a trop de répétitions, ou beaucoup... Cette typologie, si vous réussıssez à l'élaborer, me paraît prometteuse au possible...

V.I. Vous admettez donc cet espèce de divorce, chez vous, entre la méthode et la préoccupation esthétıque, une difficulté à cimenter...

G. B. Énorme. Il y a là une bâtardise incontestable. Oul, ouı... tant et si bıen qu'en classe, je le dis carrément à mes étudiants, je leur dis: "Je ne sas pas ce qui détermine la "valeur" d'une œuvre. Je sais ce que j'aime et ce que je n'aime pas et je vais essayer de vous expliquer pourquoi." Mais les raisons, les motifs viennent après coup; ce sont des espèces de ratıonalısations conscientes. Quant à une esthétique quı serait universelle, je n'y crois pas...

V. I. Elle pourrait s'élaborer à partir d'une typologie caractérielle. II y auraıt à ce moment-là possıbilité de fusion...

G. B. C'est-à-dire qu'ıl y aurait un nouveau classement d'auteurs d'imag1nation et d'œuvres d'ımagınation, un nouveau clıvage: le texte schızoïde aurait ses admirateurs schizoïdes; l'œuvre paranoïde, ses tenants paranoïdes, etc. Ensuite, viendrait l' "explication": chacun saurait qu'il n'a que la phılosophıe de ses goûts. On commence par sentır, on ne pense qu'en second lieu. Oul, votre typologie apporterait des lumières pré- 
cieuses. Elle me paraît susceptible de révolutionner la critique. J'espère que vous allez publier des choses là-dessus bientôt.

Moı, ce que j'essale de faire maintenant, c'est de m'éloigner de la psychocritique pour revenir aux anciennes distinctions entre le type visuel, auditif et verbo-moteur. Je pense que ça aussi, ça pourrait être intéressant.

V.l. Et vous, comment fantasmez-vous?

G. B. MoI, j'entends plutôt une voix. Je serais donc un «auditif».

V.I. Cette voix, vous la transcrivez au stylo?

G. B. Oul, là je me fais verbo-scripteur. Mais je ne me parle pas. Surtout je ne vois rien. Je suis aussi peu visuel, je pense, qu'on peut l'être.

V.I. Suivant l'affiliation de vos romans, on pourrait dire que le texte s'est transformé suivant une ligne directrice, à savoir la dépersonnalisation progressive de JE; c'est très évident si l'on considère la série : le Libraire, la Commensale, l'Incubation. Alors le Cycle est très surprenant puisqu'on y trouve sept JE fortement personnalisés, même s'ils sont pluriels.

G. B. OuI, mais ce sont des JE polarisés qui renvoient à un autre MOI, des JE partiels ou parcellaires en quelque sorte...

V.I. Sur le plan narratif, d'accord; mais sur le plan de l'écriture? Dans le Cycle, Il y a toujours une personnalité qui imprime son mouvement à l'écriture?

G. B. Le polarisateur. S'll y a un JE constant, s'il y a un MOI constant, c'est le cadavre, le père mort. Ce que vous dites, c'est fort possible si on se place seulement au point de vue de ces sept personnages et qu'on oublie le centre qui est le mort.

Un problème qui me préoccupe, tant au plan ındividuel que collectif, c'est l'image du père, le Père avec un grand $P$; ce que j'étudie dans mes cours, en plus des fluctuations pronominales, c'est le phénomène des "voix». Menaud est sans doute l'exemple le plus évident - qui réentend les voix de Marıa Chapdeleine. Mais j'ai retrouvé des voix analogues dans l'Appel de la race, dans Une de perdue, deux de trouvées, etc. Dans ce dernier roman, l'ıdéalisation ou la sublimation du Père est fort bien décrite. Le héros (qui souffre d'orphélinisme) découvre qu'il est le fils de son père seulement après la mort de celui-ci. Tout ce qui reste de son père, c'est un mémoıre posthume, c'est-à-dire une voix d'outre-tombe.

On retrouve un peu partout des voix qui sont à la fois particulières et collectives; la voix qu'entend Menaud, c'est celle des ancêtres, si vous 
voulez, mais c'est aussı la voıx de Louis Hémon, d'un Français, donc d'un ancêtre au plan collectif. Ça aussı c'est une questıon que j'aimerais pouvoir clarifier.

V. I. Et dans Kamouraska? II y a une voix de la conscience?

G. B. Ouı, parce qu'ıl y a des vocatifs où l'héroïne se parle à elle-même. Mais ie n'al pas encore étudié Kamouraska du poınt de vue des volx: peut-être que j'y viendraı. Le phénomène qui m'a frappé jusqu'à présent, c'est que cette héroine, sans le savoir, falt ce que sa mère a fait. Sa mère a épousé un noble et ce noble est mort. Elle, épouse un noble et le tue. Ça suit assez exactement le «pattern » maternel.

V.I. On ne saıt presque rıen de la mère d'Élısabeth, nı de son père. On en sait davantage sur les trois petites tantes.

G. B. Ouı, sauf que pour la petite à l'état fœtal, l'abandon (la mort) du père a faıllı causer sa mort et elle a une dent contre les hommes. La page 51 de Kamouraska contient un fantasme intra-utérın essentiel à la compréhensıon du caractère d'Élısabeth.

\section{V.l. Et qu'est-ce qui arrive aux Anthropoïdes?}

G. B. Pour le moment, ıls ne bougent guère. Maıs dès le mors de maı ou juın, je m'y remettraı. J'aı maıntenant au delà de 2000 pages manuscrites, peut-être, 2500 .

\section{V.l. Est-ce que c'est un récit déjà structuré?}

G. B. Il faut que je m'y remette. Je n'ai pas réussı, au début de l'année scolaıre, à le relıre. J'aı arrêté ma lecture pour me mettre à recorrıger. J'étais désespéré d'être oblıgé de retourner à mes cours. Fınalement, j'al pu ébaucher une troısième versıon partıelle. II faudrait peut-être toute l'année prochaine pour me purger de ces ancêtres.

V.I. Mais quand vous dites 2000 pages, c'est 2000 qui font le total de plusieurs versions?

G. B. C'est 2000 pages de texte survi; autrement, ce serait 3000 pages et plus.

V.I. Vous allez donc avoır à faire un travall de réduction, de réorganisation, puis de polissage terminal?

G. B. Oui, Il faudra qu'il y ait des réductions sûrement. C'est quelque chose qui m'inquiète un peu cette énormité. II s'agıt d'un monstre préhistorique: c'est le cas de le dire!

V.I. Vous avez fait des recherches assez précıses? 
G. B. Oul, J'aı lu énormément d'anthropologie et de zoologie: toutes les enquêtes sur le terraın sur lesquelles je pouvais mettre la maın. Je lısaıs ça avec passion, des foıs.

\section{V.l. Ce sera votre grande œuvre?}

G. B. La grosse, en tous cas!

L'organisation simienne me fascinait, surtout celle des macaques du Japon: une organisation oligarchique étonnante; et héréditaıre, c'est ça, au fond, qui me fascinait. Ces macaques sont gouvernés pas un triumvirat, ou par un "triumsimien", sı vous préférez. Les femelles aussi sont hiérarchisées. Il y a une femelle Alpha, une femelle Bêta, Gamma, etc. tout comme chez les mâles. Et vorci le phénomène étonnant: les petits d'une femelle Alpha ou Bêta ont de grandes chances de faire, plus tard, partie du noyau olıgarchique; alors que le fils d'une macaque "roturière" n'a à peu près aucune chance... II y a donc les mâles Alpha, Bêta, Gamma qui forment le triumvirat simien. Alpha est plus fort que Bêta et Gamma séparément, mais quand il devient trop dictatorial, Bêta et Gamma se liguent contre lui. Il y a là un noyau que j'ai essayé de reproduire chez mes Anthopoïdes...

V.I. Donc vous racontez une histoire qui se déroule au temps de nos ancêtres bien lointains?

G. B. Oui, à la fin du tertiaire.

V.I. Quı tourne autour du problème d'autorité, de prise en charge de l'oligarchie?

G. B. Oui, il y a aussi cette olıgarchie.

V.I. Ça me paraît très neuf dans vos textes, ce thème du pouvoir.

G. B. Oui et non. Mes héros d'antan n'essayaient pas de saisir le pouvorr, mais par contre ils luttaient contre lui. Dans le Libraire par exemple, Jodoin lutte contre Chicoine et contre le curé. Dans les Pédagogues, Pellerin lutte contre le directeur de l'école et contre le ministère. Dans les Anthropoïdes, j'ignore ce que ça va donner. Au début, je voulais que mes personnages ne soient pas doués de la parole. Ça n'a pas marché. Il y aura un mélange de soliloque et de narration à la première, deuxième et troisième personnes, sans parler des fantasmes collectifs parce que justement l'âme de la horde n'est pas encore formée, de même que le MOI de ces hominiens ne s'est pas encore développé comme le nôtre. Ce qui me préoccupe principalement, c'est la naissance du sur-moi, qui est cette voix intériorisée.

V.I. Comment se fait-il que tout d'un coup, vous vous intéressiez aux puissants alors que la plupart de vos personnages sont des marginaux ou des rejetés, ou des gens qui s'exilent?

G. B. Vous savez, le simple fait de créer (verbe démiurgique) une œuvre d'imagination est la manifestation d'une volonté de puissance (subli- 
mée ?). Quant à la volonté de puissance tout court, il est possible qu'elle soit en train de remonter à la surface. Pour la première fois de ma vie, je fais partie d'un conseil d'administration, celui des éditions Quinze. Alors qu'à l'Université, je me suis toujours arrangé pour les éviter. Non, une fois, j'ai accepté de faire partie d'un comité, lorsqu'il a été question de mettre sur pied le bac en Études canadiennes. Là je me suis porté volontaire. Le doyen m'a regardé, tout étonné, parce que je n'avais jamais, auparavant, accepté une telle charge. Dans le temps, cela a dû nuire à mon avancement. Je m'en suis tiré, comme Jodoin, par l'écriture. De guerre lasse, on ne m'a plus incité à faire partie de comités qui sont chronophagiques au possible.

V.I. Vous mettez de l'avant la question du Père. C'est d'ailleurs un personnage omniprésent dans vos romans; vous la voyez aussi dans une perspective qui déborde vos propres textes. Cette question est-elle liée à la naissance des Anthropoïdes?

G. B. Je pense. Les voix, le sur-moi, le Père, l'apparition du JE, les fluctuations de ce JE dans le roman, tout cela, c'est la même préoccupation.

V.I. Et l'envers de la question: avez-vous été un tenant de la thèse de Sr. Marie-Éleuthère ${ }^{6}$, cette sorte de mythologie qui avait séparé le couple, proposant un archétype maternel, et avait rejeté complètement dans l'ombre l'image du père? Avez-vous été un adepte de cette vision?

G. B. La mère est beaucoup plus évidente, je crois. Le père était absent. Il était partiellement remplacé par le père religieux, celui qu'on appelait "Mon Père", "Notre Saint-Père le pape". Le père était souvent absent; il s'en allait bûcher comme les personnages des chansons de Vigneault; il s'en allait dans les pays d'en-haut. Mais ça ne veut pas dire qu'il n'était pas intériorisé. Et comment l'intériorisation s'est-elle faite? Chez nous, je pense que c'est un problème extrêmement complexe. Vous êtes peutêtre la première génération qui avez quelques aînés intellectuels. Avant ma génération, il n'y avait pas d'intellectuels, ni tellement de créateurs. Alors nous avions toujours l'impression de recommencer à zéro. Et c'est pour ça que nous sautions l'Atlantique continuellement; nous nous reportions à la France qui est notre grand-mère, pas notre mère. Est-ce que nous sommes en train, enfin, d'avoir des liens normaux?

V.I. Vous en venez à parler de problèmes collectifs. Il semble que depuis quelques années, votre vision du Québec se particularise, se précise. Lorsque vous étiez jeune, vous alliez davantage vers Paris, plus que vers Montréal. Ne croyez-vous pas que depuis cinq ou dix ans, il commence à exister autre chose au Québec? Quelle est votre vision du Québec? Vous sentez-vous québécois?

G. B. Oui, je me sens québécois, mais peut-être pas complètement. Je me sens un peu étranger quand même, parce que la moitié de ma vie maintenant s'est passée hors frontières. J'ai quitté le Québec à vingt-six ans. J'en ai cinquante-cinq. J'ai passé trois ans au Québec (deux en sabba- 
tique, une à Laval). Je ne sais si je me sentirais moins étranger si je n'avais pas quitté le Québec. Bien des Canadiens français de ma génération (il n'y avait pas de Québécois à l'époque) qui n'ont jamais quitté "Terre-Québec" se sentent aussi... mettons un peu déphasés...

V.I. Et vous séjournez beaucoup plus à Montréal qu'auparavant.

G. B. Avant, j'étais beaucoup plus loin; en effet, je viens plus souvent. C'est le phénomène de l'involution qui se dessine peu à peu!

V. I. Vous commencez à vous "intégrer " - pour reprendre un de vos mots - dans une collectivité que vous avez contribué à faire, en tant qu'intellectuel ou en tant qu'écrivain?

G. B. J'aimerais apporter une contribution au problème du sur-moi collectif. Essayer de débroussailler la question du Père. Père biologique ou substitut paternel. Dans l'Appel de la race, ce qui est intéressant, c'est que les parents immédiats on ne les voit pas; le héros n'est sensible qu'à la lignée. II entend des voix, mais ce sont des voix d'outre-tombe, la lignée des ancêtres. Le père et la mère, il n'y en a pas.

C'est précisément parce que nous avons annihilé le père et la mère que nous avons ces voix, voix collectives et ancestrales. Alors que les parents que nous avons ne semblent pas tout à fait réels, si l'on peut dire.

V.I. Est-ce que le surmoi se rapproche de l'idéologie dominante? Quelle est la place réelle du père, puisque vous, vous parlez d'absence? Pourrait-on parler du père comme d'un contestataire, celui qui nie le discours officiel tenu par le sur-moi, celui qui s'enfuit?

G. B. Le fait que le père était en général un ignorant, un non-instruit avait pour conséquence que le dialogue et la discussion, au plan intellectuel, étaient impossibles. Et il n'y avait pas, non plus, de substitut canadien possible auparavant. C'est sans doute en train de changer. Ferron pour plusieurs est une sorte de père - un peu bizarre - mais quand même...

\section{V.I. Et Borduas?}

G. B. Oui, peut-être un peu. Mais il s'agissait d'un bien petit cénacle. D'ailleurs nous n'étions pas prêts à devenir surréalistes. Tout ce mouvement, tout ce recours à l'irrationnel pour combattre ici une intellectualité inexistante m'ont toujours paru stériles, déréalisants.

V.I. Donc, pour vous cette question du sur-moi, de l'absence du Père, apparaît comme une problématique malheureuse?

G. B. Malheureuse parce qu'on a tellement écrit de livres d'Histoire alors qu'il n'y avait pas d'Histoire. L'Histoire véritable, c'est quand on se succède de génération en génération. Pour contester la génération précédente, il faut d'abord qu'elle existe. II faudrait aussi trouver des substituts parentaux sur place et non pas recourrir à des substituts grandsmaternels ou grands-paternels comme les penseurs français et étran- 
gers. Je trouve navrant et aberrant que certains jeunes s'entichent chez nous, aujourd'hui, de Todorov, Kristeva et autres abstracteurs de quintessence telqueliens...

V.I. Est-ce que vous rattachez cela à l'absence de pouvoir politique chez les Canadiens français? Leur domination depuis trois cents ans?

G. B. Ça a dû jouer. Une partie du sur-moi provenant de l'intériorisation du conquérant. Seulement ce n'est pas là-dessus que je suis en train de travailler parce que je ne me sens pas tellement compétent dans ce domaine. La politique m'est encore très mystérieuse. II est certain que ça a joué mais il n'y a pas que ça... Ce n'est pas à cause du conquérant que nous n'avons pas voulu nous instruire davantage.

$\mathrm{Ni}$ en histoire, ni en politique, je ne me sens capable d'apporter du nouveau. Si j'apporte du nouveau, ce sera dans le domaine littéraire et dans le domaine psychique et psychologique, à cause de ces "voix».

Pour le Cycle, je me suis demandé si je ne devais pas faire le monologue intérieur du mort. Ç'aurait été conforme à la tradition (Crémazie)... je le disais en blaguant, mais quand même ça ne m'aurait pas déplu d'essayer...

V.I. Que pensez-vous de l'enseignement de la littérature à l'université ?

G. B. Il devrait y avoir beaucoup moins de cours et ils devraient être le résultat de longues recherches. Dix heures de cours magistral par année devraient suffire; suivies de discussions qui exigeraient des lectures massives de la part des étudiants. En Ontario et au Québec, la situation est la même: les étudiants ne lisent plus. Un étudiant de la dernière année de bac spécialisé m'a dit récemment: "Je suis un illettré»; et c'était vrai.

V.I. Vos objectifs à vous comme enseignant, quels sont-ils?

G. B. Des fois justement, on réussit à devenir un substitut paternel pour certains et ça peut devenir fécond à ce point de vue-là. Autrement on pourrait dire aux étudiants: "Lisez ce que j'ai publié et vous n'avez pas besoin de venir en classe. "La présence physique du professeur à l'université est trop requise. Il est victime d'une tradition antérieure à l'imprimerie. À cette époque-là, bien sûr. les cours nombreux et suivis étaient indispensables. Faute d'avoir des livres, les étudiants prenaient des notes, ils écoutaient des "lectures". (Comme vous le savez, le mot cours se traduit en anglais par lecture, précisément.) Mais aujourd'hui ça n'a plus de sens. On nous force à devenir des machines à paroles (superflues), des spécialistes du délayage... 
V.I. Comment alors verriez-vous l'intégration de l'université dans le milieu?

G. B. Je verrais une collaboration plus étroite entre les facultés des Lettres et les éditeurs en ce sens que s'il existait des séminaires de création, les résultats devraient déboucher à une maison d'édition. Le directeur ou un membre du comité de lecture d'une maison d'édition devrait être invité à ces séminaires pour dire comment se fait le tri, pour que les gens ne se sentent pas en serre chaude. C'est un projet qui me tient à cœur énormément. Maintenant je vois bien que ça ne se fera pas parce que je n'enseigne pas au Québec.

\section{V.I. Pourquoi?}

G. B. La réponse est simple: on ne m'a jamais offert un poste qui soit le moindrement acceptable, une situation qui me permette de continuer ma production. Il serait quand même loufoque que je revienne ici en sachant que je ne pourrais écrire...

V. I. Parmi les parutions récentes, quel roman québécois vous a particulièrement intéressé?

G. B. L'Hiver de force, entre autres, m'a beaucoup plu. Ça me paraît beaucoup plus réel que les autres Ducharme.

1. “L'Auteur et son œuvre. Brève rencontre avec Gérard Bessette», dans G. Bessette, le Libraire, présenté et annoté par J. Allard, Montréal, Éditions du Renouveau pédagogique, “Lecture Québec", 1970, p. 21.

2. R. Robidoux, "le Cycle créateur de Gérard Bessette ou le fond c'est la forme", dans Livres et auteurs québécois 1971, Montréal, Jumonville, 1972, p. 11-28.

3. Par exemple dans "Gabrielle Roy", dans Une littérature en ébullition, Montréal, Jour, 1968, p. 219-310.

4. "Hubert Aquin et le jeu de l'écriture", entrevue par Anne Gagnon, dans Voix et images, I, 1, septembre 1975, Montréal, PUQ, p. 5-18.

5. Allusion au passage des pages 127-129 dans la Commensale, Montréal, AS/Quinze, 1975.

6. Sr. Sainte-Marie-Eleuthère, la Mère dans le roman canadien-français, Québec, PUL, «Vie des lettres québécoises ", $n^{\circ} 1,1964$. 